D.T. Wickramasinghe, L. Ferrario, and G.V. Bicknell, eds.

\title{
Mid-infrared Observations of Magnetic Fields in the Disks of Bi-Polar Outflow Sources
}

\author{
Craig H. Smith
}

School of Physics, University College, ADFA, Canberra, ACT, 2600

Christopher M. Wright

MPIE, Postfach 1603 D-85740, Garching, Germany

David K. Aitken

Div. of Physical Sciences, University of Hertfordshire, Hatfield, UK

Patrick F. Roche

Dept. of Astrophysics, Oxford University, Oxford, OX1 3RH, UK

\begin{abstract}
We present the results from mid-infrared spectro-polarimetric observations of a number of bi-polar outflow sources. The specto-polarimetric data provides information on the polarization mechanism and the magnetic field direction. The field direction in the disks of the observed sources is most often normal to the ambient field direction and lies in the plane of the disk, indicating a toroidal rather than poloidal field configuration.
\end{abstract}

Spectropolarimetry between 8 and $13 \mu \mathrm{m}$ provides information on the chemical and physical nature of dust grains, and also on the direction of the transverse component of an aligning ambient magnetic field. (ie. the mid-infrared polarization shows the magnetic field direction, even if not its strength).

We present the results of mid-infrared polarimetric observations of $10 \mathrm{bi}-$ polar outflow sources. These wavelengths are largely free from effects of scattering, and the spectro-polarimetric data allows the separation of contributions from emissive and absorptive polarization, though absorptive polarization dominates in these heavily obscured sources. The field directions found by this method, along the line of sight to a number of protostars and young stellar objects (bi-polar outflow sources), are compared with the directions of molecular outflows and the orientations of the disc- or toroid-like structures associated with the sources.

There is a strong correlation (see Figure 1) between the local magnetic field direction (from mid-infrared polarimetry) and the orientation of the disc-like structures (CO observations from the literature). The magnetic field is found to lie in the plane of the disk, (ie. a toroidal field structure) rather than threading the disk (ie. poloidal field structure). 


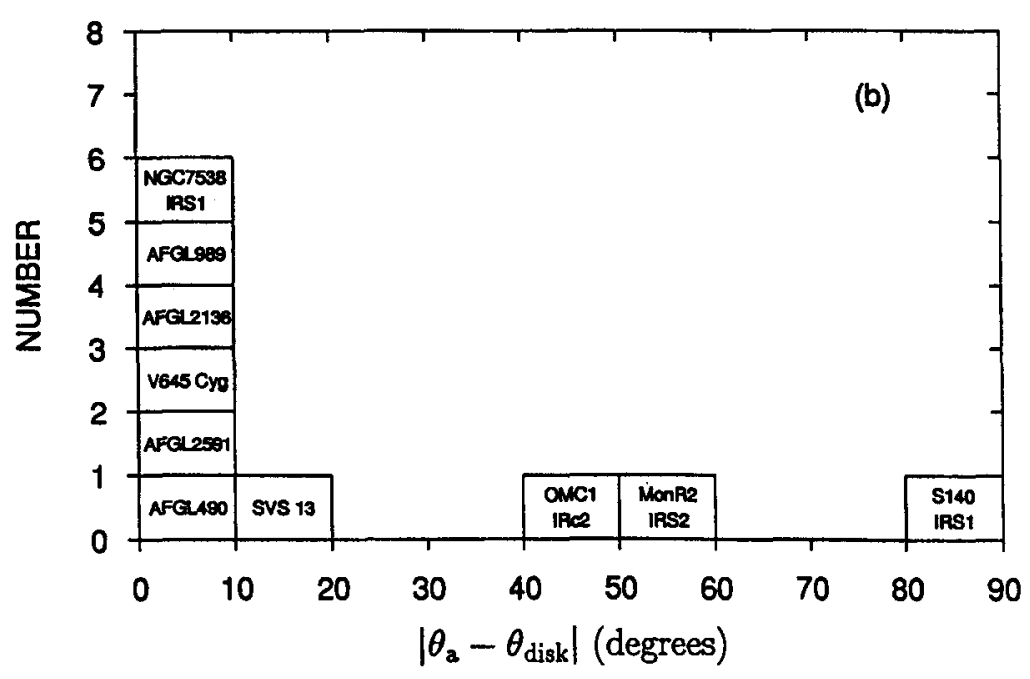

Figure 1. The mid-infrared absorptive polarization position angle,

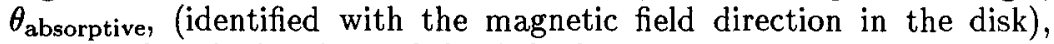
compared with the plane of the disk, $\theta_{\text {disk }}$.

In some cases (ie OMC1 IRc2, and MonR2 IRs2) spatial complexity complicates matters. We have begun a program of mid-IR imaging polarimetry to determine the magnetic field morphology in some of these sources. 